\title{
Measuring microbial growth in damp buildings: Ongoing efforts at an age-old public health problem
}

\author{
Rachel I. Adams* \\ University of California, Berkeley, USA; California Departtment of Public Health, Richmond, USA
}

\begin{abstract}
For over 2000 years, building dampness has been considered harmful. Through epidemiological efforts, we have established that damp or moldy building materials are linked to increased risk of respiratory problems. While this is presumably because of unhealthy microbial emissions, the specific causative agent(s) responsible for the health effects remain unknown. In order to identify damp buildings, we want to identify a consistent signature of microbial growth in buildings that can be detected among the rich microbial milieu normally present. This talk will provide a summary of the state of the field (hopefully addressing some long-standing misconceptions) and present findings on promising new strategies, including using sequence-based technologies, for identifying microbial growth in damp buildings. Current research on the moisture conditions in buildings under which microbes are active and contribute to volatile chemistry will also be presented.
\end{abstract}

Peer-review under the responsibility of the organizing committee of the ICMB21.

Keywords: DNA; indoor microbiome; moisture; mold; respiratory health

\section{Background}

The Book of Leviticus in the Bible, thought to be completed in $400 \mathrm{BCE}$, offers regulations and guidance about how to handle "defiling molds" on fabrics and in homes. Today, we still acknowledge the problems that dampness in buildings can bring - both to the building structure itself and to occupant health. Yet, the search remains for a consistent microbial signature in buildings that indicates moisture damage, as well as for the particular microbial product, whether biological particles or chemical emissions or something undiscovered, that induces the harmful health effect.

\section{Health effects}

Living or working in damp or moldy buildings increases the risk of many harmful health problems, including: asthma exacerbation in people who already have asthma, a new asthma diagnosis, respiratory infections (such as bronchitis), breathing symptoms (such as hay fever, sneezing, stuffy nose, sore throat, wheezing, breathing difficulty, or cough), and eczema or skin rash [1-2]. Damp or moldy buildings are associated with both allergenic and non-allergenic health responses. The associations between environmental exposure to damp or moldy buildings and respiratory health is based on epidemiological studies.

\section{Microbial measurements}

Simple observational indicators of dampness from inspection of buildings are strongly linked to respiratory health risks. These signs of dampness or mold that have scientifically demonstrated to be associated with health effects are:

- $\quad$ visible mold (regardless of color)

- mold odor

- visible water damage

- damp or moist materials

Paradoxically, measurements of specific microorganisms or microbial products have only weak, inconsistent links with health effects [3]. In fact, tests that identify the types of mold or the amounts of mold in buildings have not been demonstrated to be useful in informing health risks to building occupants (or whether any building remediation resulting from water damage was successful).

\footnotetext{
*adamsri@berkeley.edu
} 


\section{ICMB21}

\section{Ongoing efforts}

Compared to older technologies that are targeted to specific taxa or that are labor intensive, new sequence-based technologies capture the broad microbial community with high efficiency. Applications of these tools to indoor environments show that the fungal and bacterial composition of indoor environments is diverse, varying with geographic location, season, and, especially in the case of bacteria, with occupant presence and activity [4-5]. The challenge is to identify a consistent microbial signal of moisture damage within the rich diversity naturally present in buildings. Ongoing research efforts show some promise. In one approach, researchers compare indoor to outdoor samples, but with new sequence-based approaches and analytical tools [6]. Another method showing promise is examining the abundance of targeted groups of fungi, based on a priori hypothesize about their likelihood of growing in damp buildings, in damaged homes compared to dry homes [7].

In addition to particular microbial species, volatile chemicals are another potential exposure from growth in buildings. While fungi and bacteria growing on damp building materials are known to produce a suite of volatile chemicals [8], the concentrations of these compounds in actual buildings is lower than levels reported to induce health effects [9]. New technological advances allow for the detection of a broader suite of chemicals in real-time, and application of new instrumentation in indoor environments shows that biologically derived volatiles contribute to the overall chemical profile of buildings. Further work explores the specific moisture conditions and the relative influence of building material type and microbial species on influencing microbial volatile organic compounds.

\section{References}

[1] Mendell M. et al. (2011). Respiratory and allergic health effects of dampness, mold, and dampness-related agents: a review of the epidemiologic evidence. Environmental Health Perspectives, 19(6), 748-756.

[2] Kanchongkittiphon W. et al. (2015). Indoor environmental exposures and exacerbation of asthma: an update to the 2000 review by the Institute of Medicine. Environmental Health Perspectives, 123(1), 6-20.

[3] Mendell M. \& Adams R. (2019). The challenge for microbial measurements in buildings. Indoor Air, 29(4), 523-526.

[4] Adams R. et al. (2011). Dispersal in microbes: fungi in indoor air are dominated by outdoor air and show dispersal limitation at short distances. ISME Journal 7(7), 1262-1273.

[5] Barberán A et al. (2015). The ecology of microscopic life in household dust. Proceedings of the Royal Society B: Biological Sciences 282(1814), 20151139.

[6] Hegarty, B. et al. (2020). DNA Sequence-Based Approach for Classifying the Mold Status of Buildings. Environmental Science \& Technology, 54, 1596815975.

[7] Adams R. et al. (2020). Fungal signature of moisture damage in buildings: identification by targeted and untargeted approaches with mycobiome data. Applied \& Environmental Microbiology, 86(17).

[8] Korpi, A. et al. (1997). Microbial Growth and Metabolism in House Dust. International Biodeterioration and Biodegradation 40 (1): $19-27$.

[9] Korpi, A. et al. (2009). Microbial volatile organic compounds. Critical reviews in toxicology 39, 139-193.

[10] Adams, R et al. (2017). Microbes and associated soluble and volatile chemicals on periodically wet household surfaces. Microbiome 5, 128. 